\title{
The regulatory role of Pcf11-similar-4 (PCFS4) in Arabidopsis development by genome-wide physical interactions with target loci
}

\author{
Denghui Xing ${ }^{1,4^{*}}$, Yajun Wang ${ }^{1}$, Ruqiang Xu ${ }^{1,5}$, Xinfu Ye ${ }^{2}$, Dewei Yang ${ }^{2}$ and Qingshun Q Li $i^{1,2,3^{*}}$
}

\begin{abstract}
Background: The yeast and human Pcf11 functions in both constitutive and regulated transcription and pre-mRNA processing. The constitutive roles of PCF11 are largely mediated by its direct interaction with RNA Polymerase II C-terminal domain and a polyadenylation factor, Clp1. However, little is known about the mechanism of the regulatory roles of Pcf11. Though similar to Pcf11 in multiple aspects, Arabidopsis Pcf11-similar-4 protein (PCFS4) plays only a regulatory role in Arabidopsis gene expression. Towards understanding how PCFS4 regulates the expression of its direct target genes in a genome level, ChIP-Seq approach was employed in this study to identify PCFS4 enrichment sites (ES) and the ES-linked genes within the Arabidopsis genome.
\end{abstract}

Results: A total of 892 PCFS4 ES sites linked to 839 genes were identified. Distribution analysis of the ES sites along the gene bodies suggested that PCFS4 is preferentially located on the coding sequences of the genes, consistent with its regulatory role in transcription and pre-mRNA processing. Gene ontology (GO) analysis revealed that the ES-linked genes were specifically enriched in a few GO terms, including those categories of known PCFS4 functions in Arabidopsis development. More interestingly, GO analysis suggested novel roles of PCFS4. An example is its role in circadian rhythm, which was experimentally verified herein. ES site sequences analysis identified some over-represented sequence motifs shared by subsets of ES sites. The motifs may explain the specificity of PCFS4 on its target genes and the PCFS4's functions in multiple aspects of Arabidopsis development and behavior.

Conclusions: Arabidopsis PCFS4 has been shown to specifically target on, and physically interact with, the subsets of genes. Its targeting specificity is likely mediated by cis-elements shared by the genes of each subset. The potential regulation on both transcription and mRNA processing levels of each subset of the genes may explain the functions of PCFS4 in multiple aspects of Arabidopsis development and behavior.

Keywords: Transcription, Polyadenylation factor, RNA processing, Alternative processing, ChIP-seq

\section{Background}

Gene transcription and pre-mRNA processing are two major processes in eukaryotic mRNA biosynthesis. RNA processing events were thought to follow transcription. However, studies in the past two decades have well established that the pre-mRNA processing is highly cotranscriptional in vivo [1]. Pcf11 (Protein 1/Cleavage Factor 1 ) is one of such proteins that couple pre-mRNA processing with transcription [2-5].

\footnotetext{
*Correspondence: dxing@mail.colostate.edu; liq@miamioh.edu ${ }^{1}$ Department of Botany, Miami University, Oxford, OH 45056, USA ${ }^{4}$ Current Address: Department of Biology, Colorado State University, Fort Collins, CO 80526, USA

Full list of author information is available at the end of the article
}

Originally identified as a factor required for pre-mRNA 3 '-end processing and transcription termination $[2,6]$, Pcf11 was also eventually found to play a role in transcription initiation, elongation and mRNA export from nucleus to cytoplasm [7-10]. Recent studies revealed additional roles of Pcf11 in transcription termination of snRNA, snoRNA and cryptic unstable transcripts $[8,11]$. The effects of Pcf11 on transcription and pre-mRNA processing are largely mediated by its interactions with RNA polymerase II C-terminal domain (Poly II CTD) and other polyadenylation factors [3-5,12-15]. Disruption of these interactions led to altered transcription termination and decreased polyadenylation efficiency $[12,13]$. The interactions could be affected directly by the

\section{Biomed Central}


phosphorylation status of Pol II CTD and indirectly by cis-elements within pre-mRNA, possibly through RNAbinding factors (e.g. RNA15 and Hrp1) [6,16-21].

Given the key role of Pcf11 in coupling transcription termination and 3 'end processing, it is not surprising that Pcf11 also serves as a target for regulated transcription and pre-mRNA processing. Recent studies revealed its additional roles, direct and indirect, in regulated transcription initiation, elongation, termination and alternative processing of pre-mRNA $[7,8,10,15,22]$.

PCFS4 is one of the Arabidopsis orthologs of yeast and human Pcf11 [23]. While similar to Pcf11 in both its amino acid sequence and domain structures, PCFS4, unlike its yeast or human counterpart, was not required for the viability of Arabidopsis plants [23]. Functional characterizations revealed the role of PCFS4 in Arabidopsis development such as flowering time [23]. Molecular characterizations suggested that the function of PCFS4 in flowering control was partially mediated by the alternative processing (AP) of $F C A$, a gene encoding a flowering time regulator [23]. However, the AP of FCA could not fully account for the delayed flowering of $p c f s 4$ mutants, nor was it responsible for the other developmental defects, suggesting that there must be gene(s) other than $F C A$ being targeted by PCFS4 [23]. Supporting this hypothesis were the hundreds of differentially expressed genes in the $p c f s 4-1$ mutant revealed by genome-wide gene expression profiling [24].

Given that Pcf11 was recruited to actively transcribed gene loci through interaction with Pol II CTD $[3,25]$, we hypothesize that PCFS4 may also physically interact with the loci of its direct targets. To test this hypothesis, we employed a ChIP-Seq assay to identify PCFS4 enriched sites (ES) within the Arabidopsis genome. The results shed light on what genes were directly targeted and how PCFS4 might be recruited to the loci of those targets. Gene ontology (GO) analysis of the ES-linked genes revealed enriched GO terms that both explained the known developmental defects of pcfs 4 mutants and suggested additional regulatory roles of PCFS4 in other biological processes.

\section{Results}

PCFS4 functions in multiple aspects of

\section{Arabidopsis development}

Previously, we have genetically and molecularly characterized the functions of PCFS4 in flowering time control. We found that the flowering delay of the pcfs4 mutants could be partially explained by the effects of PCFS4 on the alternative processing of $F C A$ pre-mRNA [23]. In addition to its delayed flowering, $p c f s 4$ mutants also showed other phenotypes including reduced vigor of their seedlings, altered leaf shape and inflorescence phyllotaxy (Figure 1). In the early seedling stage,

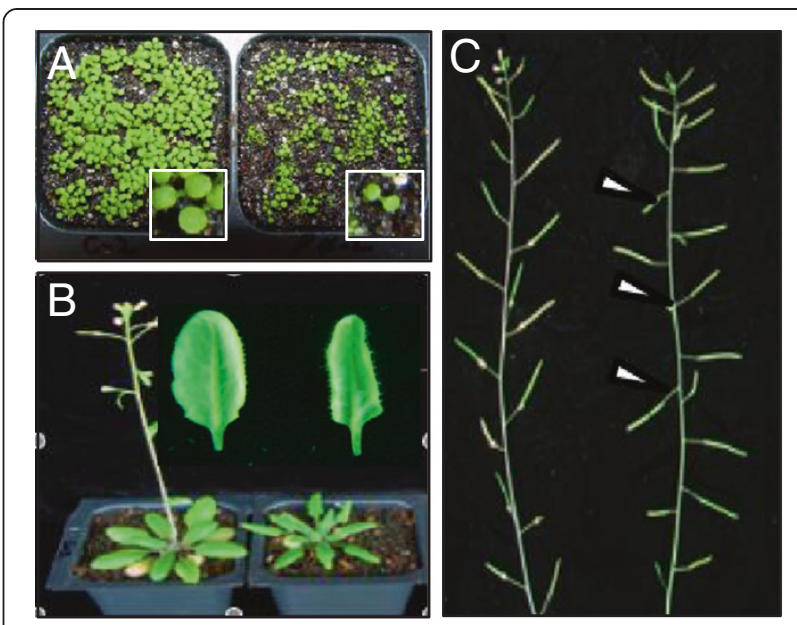

Figure 1 The pleiotropic effects of pcfs 4 mutant on Arabidopsis development. Shown on individual panels are wild type Col (left hand side) and mutant pcfs4-1 (right hand side). The mutant showed a variety of aberrant morphologies including smaller seedlings (A), down-curved leaf shape, delayed flowering (B), and altered inflorescence phyllotaxy (C). The embedded seedlings within panel A were a detailed look with equal magnification. The arrows within panel $\mathrm{C}$ point to the siliques forming a larger angle with the stem. Pictures were taken at 10,40, 50 days after germination, respectively, for (A), (B) and (C).

mutant plants were significantly smaller than the wild type (Figure 1A). Leaf edges of $p c f s 4-1$ were more curved down towards its abaxial side (Figure 1B). The siliques of pcfs4-1 mutants formed a larger angle with its stem in contrast to that in wild type Col (Figure 1C). Albeit all these defects, the mutant remained quite healthy throughout its whole life cycle in the standard growth conditions, suggesting that PCFS4 is not essential for the viability of the plants. Supporting this conclusion is that the expression of PCFS4 is not ubiquitous, but instead, tissue-specific and developmentally regulated [23]. The non-essential nature of PCFS4 for plant viability and specific developmental defects of $p c f s 4$ mutants support a hypothesis that PCFS4 might specifically target on a subset of genes.

\section{PCFS4 interacts with Arabidopsis Pol II CTD domain}

In mammals and yeast, Pcf11 is recruited to actively transcribed gene loci through its interaction with phosphorylated Pol II CTD domain $[3,25]$. The interaction plays a pivotal role in mediating the effects of Pcf11 on both transcription and pre-mRNA processing $[3-5,12,13]$. To examine whether PCFS4 interacts with Arabidopsis Pol II CTD, we carried out a modified yeast two-hybrid assay $(\mathrm{Y} 2 \mathrm{H})$ [26]. In the assay, the bait contained the Kin28-tethered Arabidopsis Pol II CTD domain. Kin28 is a protein kinase and tethering it with the CTD ensures that the CTD is phosphorylated [26,27]. The results of $\mathrm{Y} 2 \mathrm{H}$ assays indicated that PCFS4 did interact with the 
CTD-Kin28. And since no interaction was detectable when Kin28 was mutated (Figure 2), we concluded that the interaction was dependent on the phosphorylation of the CTD. To rule out that the interaction might be attributed to kin28 portion of the fusion protein, the interaction between PCFS4 and Kin28 alone was tested, with the result being that no interaction was detectable (Figure 2). Taken together, these results indicated that PCFS4 did interact with phosphorylated Pol II CTD, further supporting the idea that PCFS4 might be recruited to the loci of its direct targets.

\section{PCFS4-TAP fusion protein was enriched on hundreds of genomic regions}

To address if PCFS4 physically interact with its target genes and what these genes might be, we transformed the pcfs4-1 mutant with a transgene encoding PCFS4TAP (Tandem Affinity Purification) fusion protein. The transgene successfully complemented the mutant phenotypes and the expression of the fusion protein was confirmed by western blots using the peroxidase-conjugated anti-peroxidase antibody against the TAP tag (Additional file 1: Figure S1). We then performed a ChIP (chromatin immunoprecipitation) using the same antibody following the formaldehyde cross-linking treatment of two-week old seedlings. The ChIP DNA and the input DNA were further sequenced using Illumina sequencing platform.

The sequence reads (75-base long) derived from Illumina sequencing were first mapped to the Arabidopsis genome

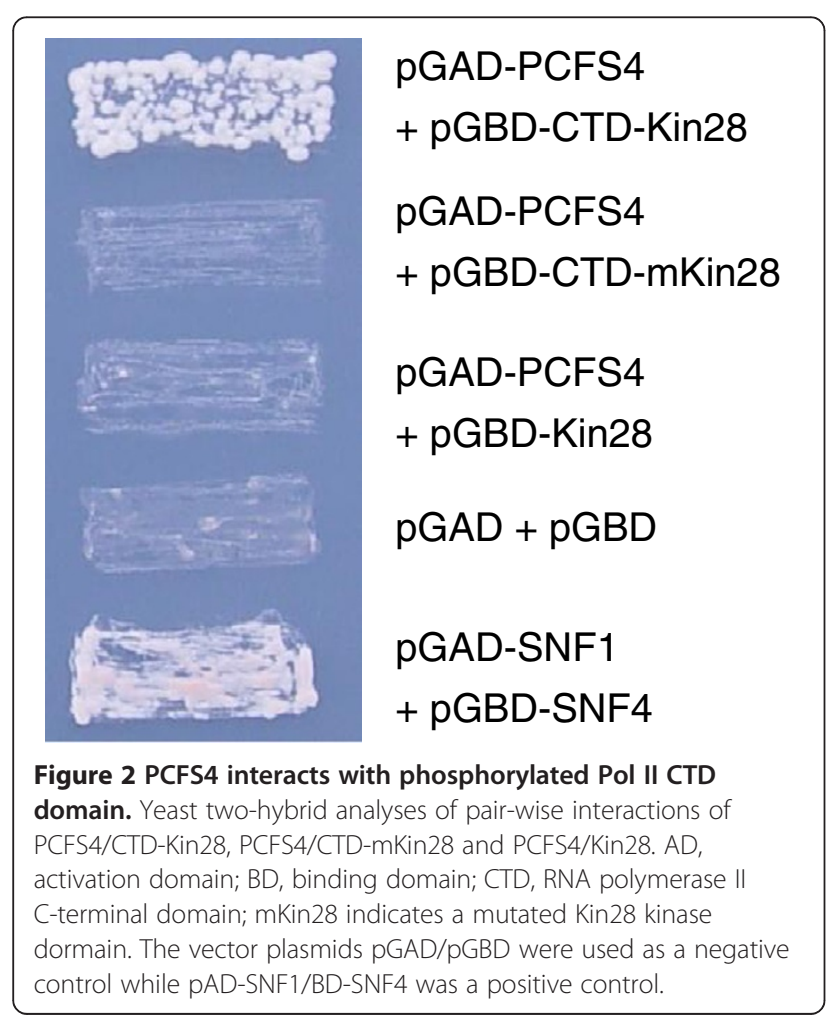

using Bowtie [28]. About 2 million and 4.2 million of reads from ChIP and input samples, respectively, were successfully mapped to the genome. The mapped reads were further analyzed using Cisgenome to identify the enrichment sites (ES) that were over-represented in the sequence reads from the ChIP sample [29]. The input sample was used as a background control in this analysis. 892 ES sites were identified with the following criteria: Log2 Fold Change $\geq 5 ; \mathrm{p}$-value $\leq 0.001$; and the false discovery rate $(\mathrm{FDR})=0.02$ (Additional file 2: Table S1).

To verify the enrichment of PCFS4-TAP on the identified ES, we performed a real-time PCR (qPCR) analysis following the ChIP for 9 randomly selected ES sites, which cover the entire ES list with p-value ranking from low to high (Additional file 2: Table S1). The results indicated that PCFS4-TAP was indeed enriched on all tested ES sites in the ChIP sample. The enrichment ranged from 2 to 5 fold, relative to the control sites (Figure 3). Thus, the identified ES were truly representing the PCFS4-TAP enrichment sites within Arabidopsis genome.

When the distribution of the ES was examined, it was found that $80 \%$ of the ES were located on intragenic regions (Table 1). For the ES on intergenic regions, the majority of them were located in the vicinity of genes. 95\% of ES were located either within genes or $1 \mathrm{~kb}$ up- or down-stream of the genes (Figure 4). Thus, PCFS4 was preferentially located on the intragenic region, consistent with its potential role in regulating transcription and pre-mRNA processing.

When the ES distribution within the intragenic region was considered, $66 \%$ of the ES were located within exons,

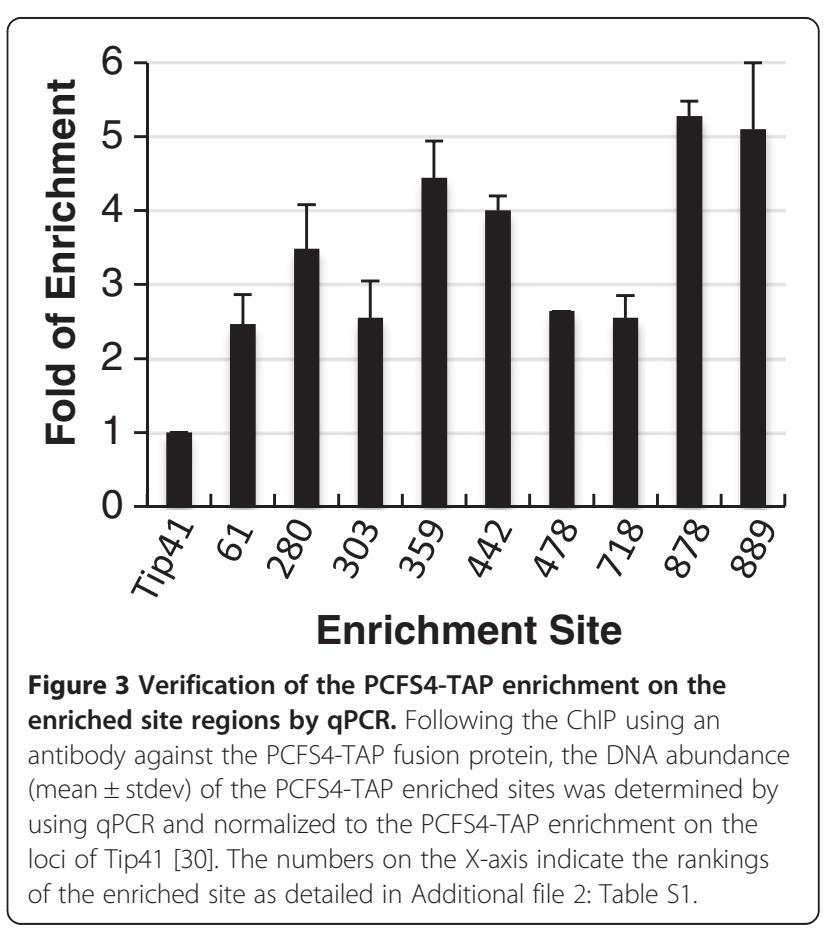


Table 1 The distribution of the enriched sites (ES)

\begin{tabular}{|c|c|c|c|c|c|}
\hline Location & & & No. ES & Subtotal ES (\%) & Total ES (\%) \\
\hline Intergenic & & & 177 & & 19.8 \\
\hline \multirow[t]{4}{*}{ Intragenic } & Exon & 5UTR & 33 & 5 & 65.7 \\
\hline & & CDS & 485 & 81 & \\
\hline & & 3'UTR & 82 & 14 & \\
\hline & Intron & & 129 & & 14.5 \\
\hline Total & & & 892 & & 100 \\
\hline
\end{tabular}

while $15 \%$ of them were located within introns (Table 1 ). Of the ES within exons, there are 5\% within 5'UTR; $81 \%$ within coding sequence region (CDS); and 14\% within 3 ' UTR (Table 1). Thus, PCFS4-TAP was predominantly enriched within CDS region.

\section{Identification of common cis-elements}

With the identified ES, we reasoned that there could exist a unique, shared cis-element(s) rendering these ES to be specifically targeted by PCFS4, either directly or indirectly [through other factor(s)]. To explore the potential cis-element(s), we analyzed the sequences of these ES using MEME-ChIP program [31]. Three sequence motifs with varied significance, ranging from $2.2 \times 10^{-33}$ to $2.0 \times 10^{-3}$, were identified (Figure 5). Motif 1 was 21 bp long and shared by 85 ES sites; motif 2 was 15 bp long and shared by 43 ES sites; and motif 3 was 11 bp long and shared by 69 ES sites (Figure 5). However, not all ES sites (892) were covered by the 3 motifs, suggesting additional motifs that could not be identified by this method.

\section{GO enrichment analysis of ES-linked genes}

To explore the biological significance of PCFS4 enrichments on the identified ES, the ES-linked genes were

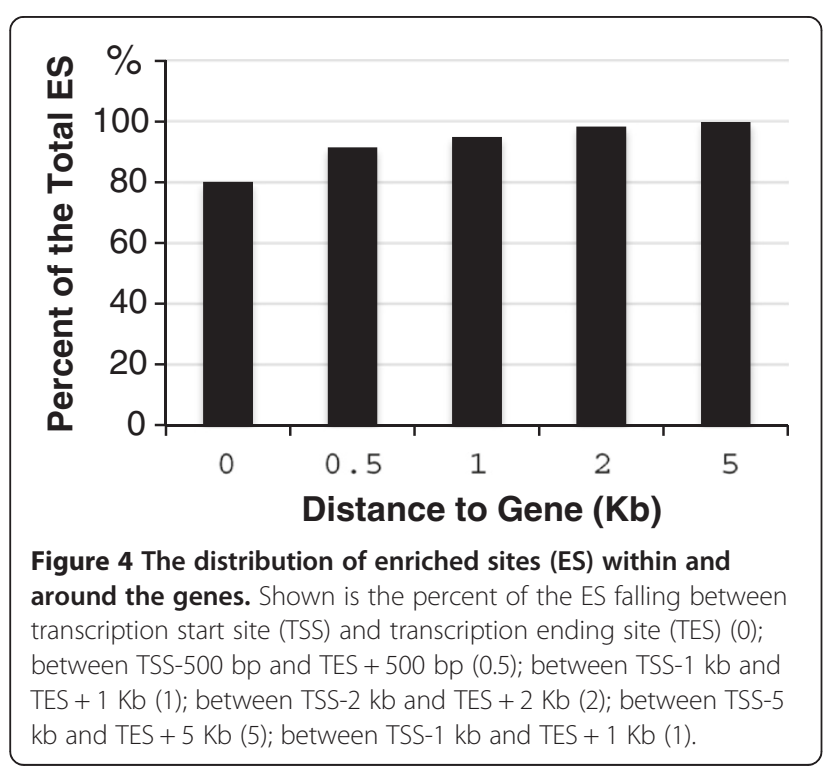

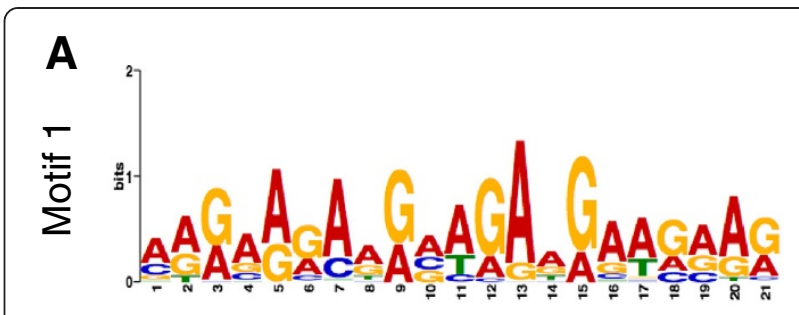
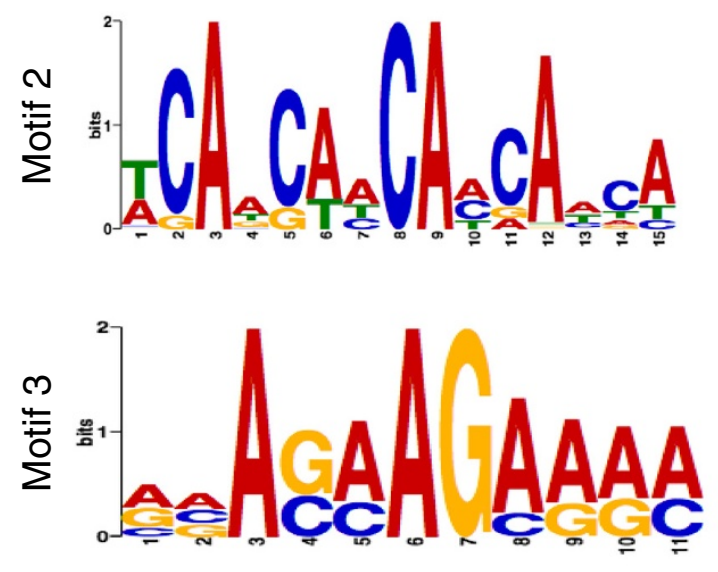

B

\begin{tabular}{|c|ccc|}
\hline Motif \# & Width & No. ES & E-value \\
\hline Motif 1 & 21 & 85 & $2.20 \mathrm{E}-33$ \\
Motif 2 & 15 & 43 & $1.80 \mathrm{E}-09$ \\
Motif 3 & 11 & 69 & $2.00 \mathrm{E}-03$ \\
\hline
\end{tabular}

Figure 5 The sequence motifs identified from ES sequences. Shown are the sequence logos of Motif 1, Motif 2 and Motif 3 (A) and their Width (Nucleotide No.), the number of covered ES sites (No. ES) and their significance level (E-value) (B).

extracted and analyzed. An ES-linked gene is defined as one that is closest to a given ES or an ES within $2 \mathrm{~Kb}$ upstream of the gene's start codon and $2 \mathrm{~Kb}$ down-stream of its stop codon. 839 such genes were identified, with a majority of them being linked with a single ES (821 or $98 \%$ ) and 18 of them (2\%) linked with 2 ES (Additional file 2: Table S1).

With the ES-linked genes as inputs, GO enrichment analyses were performed using GeneCodis [32]. The results revealed that multiple GO terms were overrepresented in the ES-linked genes (p-value $\leq 0.01$; FDR $\leq 0.05$ ) (Table 2; Additional file 2: Table S2; Table S3). A similar set of enriched GO terms was identified when the genes were analyzed using GOEAST, another GO enrichment analysis tool (Additional file 2: Table S4) [33]. Among those GO terms were "photomorphogenesis", "Embryo development ending in seed dormancy" and "negative regulation of flower development", which are consistent with the known altered morphologies and 
Table 2 Enriched GO terms identified with GeneCodis

\begin{tabular}{|c|c|c|c|c|c|}
\hline GO ID & Ontology\# & Term & $\begin{array}{l}\text { Num. of } \\
\text { Genes }\end{array}$ & p-value & $\mathrm{FDR}^{*}$ \\
\hline GO:0016049 & $\mathrm{BP}$ & cell growth & 7 & $3.4 \mathrm{E}-06$ & $3.5 \mathrm{E}-04$ \\
\hline GO:0005086 & MF & ARF guanyl-nucleotide exchange factor activity & 4 & $5.5 \mathrm{E}-06$ & $6.4 \mathrm{E}-04$ \\
\hline GO:0005488 & MF & binding & 36 & $6.3 \mathrm{E}-05$ & $2.4 \mathrm{E}-03$ \\
\hline GO:0009640 & $\mathrm{BP}$ & photomorphogenesis & 7 & $1.5 \mathrm{E}-04$ & $5.4 \mathrm{E}-03$ \\
\hline GO:0009555 & $\mathrm{BP}$ & pollen development & 11 & $2.5 \mathrm{E}-04$ & $6.4 \mathrm{E}-03$ \\
\hline GO:0016192 & $\mathrm{BP}$ & vesicle-mediated transport & 10 & $6.7 \mathrm{E}-04$ & $1.4 \mathrm{E}-02$ \\
\hline GO:0042752 & $\mathrm{BP}$ & regulation of circadian rhythm & 4 & 7.1E-04 & $1.2 \mathrm{E}-02$ \\
\hline GO:0004553 & MF & hydrolyzing O-glycosyl compounds & 16 & 7.7E-04 & $1.5 \mathrm{E}-02$ \\
\hline GO:0009793 & $\mathrm{BP}$ & embryo development ending in seed dormancy & 19 & $1.0 \mathrm{E}-03$ & $1.3 \mathrm{E}-02$ \\
\hline GO:0004672 & MF & protein kinase activity & 28 & $1.1 \mathrm{E}-03$ & $1.9 \mathrm{E}-02$ \\
\hline GO:0080167 & $\mathrm{BP}$ & response to karrikin & 10 & $1.3 \mathrm{E}-03$ & $1.4 \mathrm{E}-02$ \\
\hline GO:0050790 & $\mathrm{BP}$ & regulation of catalytic activity & 4 & $1.4 \mathrm{E}-03$ & $1.2 \mathrm{E}-02$ \\
\hline GO:0009561 & $\mathrm{BP}$ & megagametogenesis & 4 & $1.4 \mathrm{E}-03$ & $1.2 \mathrm{E}-02$ \\
\hline GO:0003700 & MF & sequence-specific DNA binding transcription factor activity & 61 & $2.1 \mathrm{E}-03$ & $3.1 \mathrm{E}-02$ \\
\hline GO:0005524 & MF & ATP binding & 52 & $2.1 \mathrm{E}-03$ & $2.8 \mathrm{E}-02$ \\
\hline GO:0003677 & MF & DNA binding & 53 & $2.9 \mathrm{E}-03$ & $3.4 \mathrm{E}-02$ \\
\hline GO:0008270 & MF & zinc ion binding & 48 & 3.7E-03 & $3.6 \mathrm{E}-02$ \\
\hline GO:0050832 & $\mathrm{BP}$ & defense response to fungus & 9 & $5.4 \mathrm{E}-03$ & 4.3E-02 \\
\hline GO:0009832 & $\mathrm{BP}$ & plant-type cell wall biogenesis & 4 & $5.6 \mathrm{E}-03$ & 4.1E-02 \\
\hline GO:0015031 & $\mathrm{BP}$ & protein transport & 8 & $6.4 \mathrm{E}-03$ & 4.4E-02 \\
\hline GO:0004871 & MF & signal transducer activity & 8 & $6.4 \mathrm{E}-03$ & $4.9 \mathrm{E}-02$ \\
\hline GO:0044237 & $\mathrm{BP}$ & cellular metabolic process & 4 & $7.9 \mathrm{E}-03$ & 4.9E-02 \\
\hline GO:0000226 & $\mathrm{BP}$ & microtubule cytoskeleton organization & 3 & $8.1 \mathrm{E}-03$ & 4.7E-02 \\
\hline
\end{tabular}

Note: \#: $B P$, Biological Process; $M F$, Molecular Function.

*: FDR, False Discovery Rate.

delayed flowering of $p c f s 4-1$ mutants (Figure 1; Table 2; Additional file 2: Table S3). The other enriched GO terms include circadian rhythm, gametophyte development, protein kinase activity, plant cell wall synthesis, and response to fungus, suggesting potential roles of PCFS4 in additional biological processes (Table 2). These results implied that PCFS4 might serve as an important regulator of transcription and pre-mRNA processing for genes involved in a variety of biological functions in plants.

\section{PCFS4 plays a role in Arabidopsis circadian rhythm}

To find further supporting evidence for the implied roles of PCFS4 in "additional" biological processes, we focused on the genes within the enriched GO term "regulation of circadian rhythm". The GO term contains 5 ES-linked genes (Additional file 2: Table S5). The enrichment of PCFS4 on these loci was verified by qPCR (Additional file 1: Figure S2). Since PCFS4 was an ortholog of Pcf11 and known to play a role in alternative pre-mRNA processing [23], we examined the alternative processing profiles of these genes using publically available cDNA/ EST data (TAIR10). Indeed, all five genes showed certain forms of alternative transcription and/or pre-mRNA processing evidenced by the cDNA and/or EST data (Additional file 1: Figure S3). Most importantly, the ES sites on these genes were often associated with the positions where the alternative processing or alternative transcription initiation occurred (Additional file 1: Figure S3).

We further tested the effects of PCFS4 mutation on circadian rhythm in Arabidopsis by quantifying the expression of TOC1 and CCA1, two key circadian clock genes [34,35]. As shown in Figure 6, both the amplitude and circadian period were altered for the expression of TOC1 and CCA1, with the pcfs 4 mutant showing a lengthened circadian period and increased amplitude for both genes. Thus, PCFS4 indeed played a role in Arabidopsis circadian rhythm, likely through direct and indirect regulation of the transcription and/ or pre-mRNA processing of its target genes. 


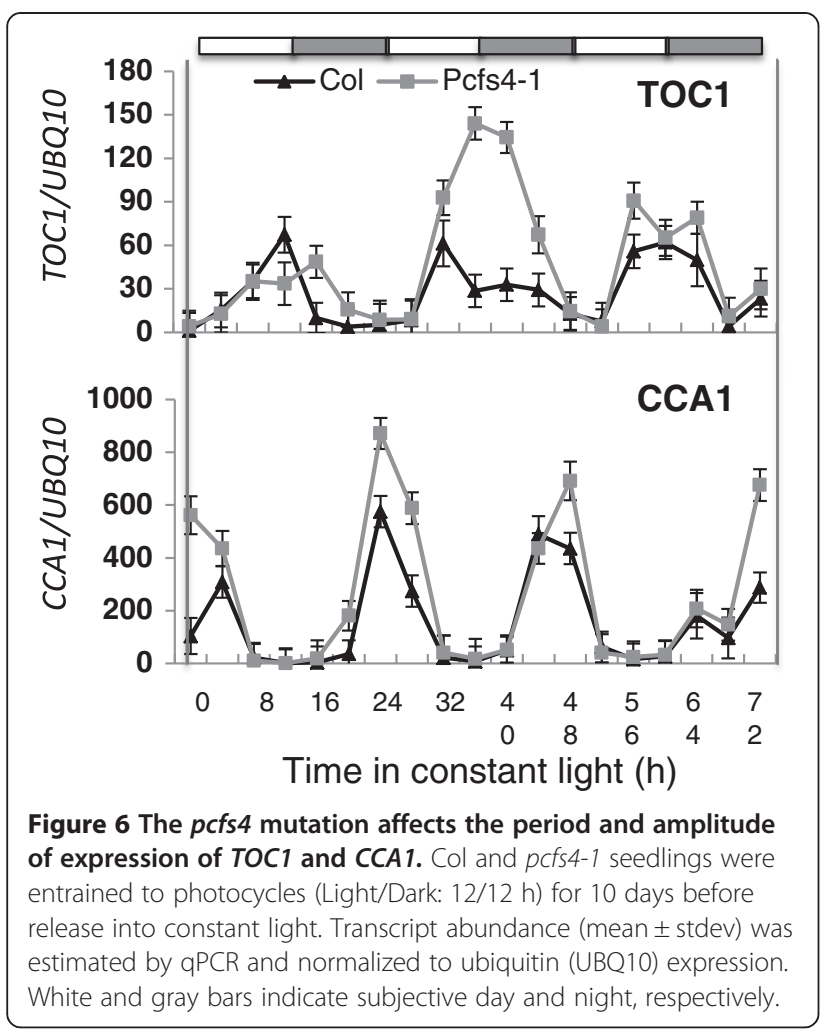

\section{Discussion}

Accumulating evidence in all three kingdoms of eukaryotic organisms supports the idea that alternative processing of pre-mRNA plays a key role in regulation of gene expression and the transcriptome complexity [36-41]. Among the many factors affecting AP of premRNA, one is Pcf11. While essential for pre-mRNA 3' end processing and transcription termination in general, there was evidence that Pcf11 played a regulatory role in the pre-mRNA processing of some genes $[15,22,42,43]$. Being one of Arabidopsis orthologs of Pcf11, PCFS4 was of special interest in that it is, unlike Pcf11, not essential for the plant viability [23]. The non-essential nature and the pleiotropic effects of PCFS4, together with its proved regulatory role in $F C A$ pre-mRNA processing, argue that PCFS4 may have specifically adapted itself for regulated pre-mRNA processing of a subset of genes in plants. However, how PCFS4, like Pcf11 when playing a regulatory role, gains its specificity remains elusive.

The interaction of PCFS4 with Pol II CTD provides evidence that PCFS4 was recruited to actively transcribed gene loci (Figure 2). This interaction, on the other hand, does not define the target genes by PCFS4 since Pol II CTD is universally required for all actively transcribed protein-coding genes. Towards understanding how PCFS4 might gain its target specificity, we identified the genome-wide PCFS4-TAP ES sites that were specifically concentrated on the genic regions (Table 1 ).
Bioinformatic analysis identified a few unique sequence motifs that were shared by some of the ES sites (Figure 5). These sequence motifs could be essential elements providing PCFS4 target specificity either as cis-elements within genes or pre-mRNAs. So, how are the cis-elementcontaining genes specifically targeted by PCFS4? One scenario could be that the cis-elements within the pre-mRNA compete with Pol II CTD for binding PCFS4 so that the interaction between PCFS4 and CTD is disrupted, leading to an altered pre-mRNA processing. Evidence supporting this scenario is the weak RNA binding activity of Pcf11 and the competitive Pcf11binding between RNA and Pol II CTD [17]. Alternatively, the cis-elements within the gene may affect the phosphorylation status of Pol II CTD, leading to gene-specific CTD code(s), which again may influence the CTD-PCFS4 interaction [18].

The predominant location of ES on the CDS region was surprising, given that Pcf11 in yeast was preferentially mapped to the 3' end of the gene loci [18-21]. However, this discrepancy might well explain the non-essential nature of PCFS4. In other words, PCFS4 may mainly play a regulatory role for transcription and pre-mRNA processing of a subset of genes while its yeast ortholog, pcf11, acts mainly as a general transcription termination and 3 'end pre-mRNA processing factor [20,21].

The GO enrichment analysis of ES-linked genes revealed the functions of PCFS4 beyond what we have known previously. Not only were the enriched GO terms consistent with PCFS4's functions in Arabidopsis development and flowering control but also revealed its potential roles in circadian rhythm, response to fungus pathogen and plant cell wall synthesis (Table 2). We were also able to verify PCFS4's effects on Arabidopsis circadian rhythm and the coincidence of PCFS4 ES sites with the sites where the alternative processing was suggested by cDNAs and/or ESTs. This shined light on how PCFS4 functions in this biological process. Interestingly, recent studies also revealed a significant role of pre-mRNA alternative processing in regulating the expression of circadian clock genes [44-47]. Our discovery offers additional evidence of such a regulation.

These results, together with what have been known about PCFS4 and Pcf11, lead to a conceivable model by which the biological functions of PCFS4 might be explained. In this model, PCFS4 is recruited to the loci of subsets of genes. Each subset of genes, whose regulated expression mediates a specific biological effect of PCFS4, shares a common cis-element. The cis-element, when existing in pre-mRNA, may affect the PCFS4-CTD interaction by competitively binding PCFS4 with CTD, or by recruiting another PCFS4-binding factor $[14,17]$. Alternatively, when present on the gene, the cis-element may recruit factors affecting the phophorylation status of 
Pol II CTD domain [18]. By either way, the PCFS4-CTD interaction will be affected, leading to altered gene transcription and/or pre-mRNA processing. Depending on the functional nature of each subset of genes, the cis-element and its relative locations on the genes ( $5^{\prime}$ end, 3 ' end or middle section of the gene) could vary. The protein factors mediating the cis-element's function may be unique for each subset of genes. The combination of the cis-elements, their locations and the mediating factors may explain the multiple biological effects of PCFS4.

\section{Conclusions}

It is demonstrated that Arabidopsis PCFS4 specifically targets subsets of genes. Its targeting specificity is likely mediated by the cis-element shared by the genes of each subset. The potential regulation at the level of transcription and mRNA processing may be the basis for its multi functions in different aspects of Arabidopsis development and environmental responses. The targeting specificity of Arabidopsis PCFS4 might also suggest a potential mechanism of human and yeast Pcf11 in regulating gene transcription and mRNA processing.

\section{Methods}

All Arabidopsis thaliana plants used in this study are in Col background. The yeast strains, the pGADPCFS4 construct and control plasmids for $\mathrm{Y} 2 \mathrm{H}$ assay had been described previously [23]. The pGBD-CTDKin28, pGBD-CTD-mKin28, and pGBD-Kin28 were kind gifts from Dr. Hisashi Koiwa (Texas A\&M University). The $\mathrm{Y} 2 \mathrm{H}$ assay was performed as described previously [23].

Arabidopsis seeds were germinated and grown on Sun Grow 360 soil under standard conditions as described previously [23]. Plant pictures were taken at different growth stages suitable for each phenotype as indicated.

The ChIP assay was carried out largely based on the published protocols with slight modification $[48,49]$. Briefly, Transgenic seeds containing PCFS4-TAP transgene were germinated on MS medium at $4^{\circ} \mathrm{C}$ in dark for 2 days and then moved to a grow chamber with $22^{\circ} \mathrm{C}, 16 / 8 \mathrm{hr}$ light/ dark cycles. Two-week old seedlings were harvested and cross-linked with $1 \%$ formaldehyde and further processed as described [48]. The chromatin was sheared by sonication to $300-1000$ bp fragments. The sample was centrifuged and the supernatant was transferred to two siliconized tubes, one for immuno-precipitation (IP) and the other as an input control (IN). For the IP sample, $60 \mu \mathrm{l}$ sepharose IgG beads was added and incubated for $3 \mathrm{hr}$ at $4^{\circ} \mathrm{C}$. The sample was washed and the bead-binding complexes were eluted with elution buffer [48]. The IN sample and the eluted IP sample were treated with 200
$\mathrm{mM} \mathrm{NaCl}$ to reverse the cross-linking. The samples were digested by proteinase $\mathrm{K}$ to remove proteins, treated with phenol/chloroform extraction, and DNA fragments were recovered by ethanol precipitation. The enrichment of DNA on tested genomic regions was estimated using real-time PCR (qPCR). The oligonucleotide primers used for detecting each ES site or ES sites within genes were listed in Additional file 2: Table S6.

For the ChIP-Seq assay, the ChIP samples were prepared essentially the same as described above except that the chromatin was sheared to 100 to 500 bp long fragments. The precipitated DNA was further processed following the instruction of Illumina ChIP-Seq DNA Sample Prep Kit (Illumina Inc). The DNA library was sequenced using Illumina platform Genome Analyzer II in the Ohio State University MCIC (Wooster, Ohio). The sequencing reads were mapped to the Arabidopsis genome (TAIR10; www. arabidopsis.org) using Bowtie with the following mapping parameters: the quality matrix, phred64-quals; the minimum seed length, $l=20$; the allowed mismatch in the seed, $n=1$ [28]. The mapped reads (both IP and IN) were used as input to analyze the significantly enriched peaks using Cisgenome with default parameters [29]. The sequences of the enriched peak sites and their linked genes were extracted using the same program package. The sequence data (.fastq files) were deposited to The NCBI Sequence Read Archive with accession number of SRA060798.

For sequence motif identifications, the enriched peak sites were analyzed using MEME-CHIP to find overrepresented sequence motifs with the parameter setting: Distribution of motif occurrences, zero or one per sequence; Minimum motif width, 6; Maximum motif width, 30 [31]. For the GO enrichment analysis, the ES-linked genes were analyzed using GeneCodis and GOEAST program packages [32,33].

For circadian rhythm analysis, the seeds of $\mathrm{Col}$ and pcfs4-1 mutant were germinated on MS medium at $4{ }^{\circ} \mathrm{C}$ in the dark for 2 days and moved to growth chamber under $22^{\circ} \mathrm{C}, 12 / 12 \mathrm{hr}$ light/dark photoperiod. Ten days later, the chamber was set on constant light conditions. The seedlings were collected every four hours starting right at the beginning of the constant light and ending at $72 \mathrm{hrs}$. The collected seedlings were immediately frozen in liquid nitrogen and stored at $-80^{\circ} \mathrm{C}$ until all the samples were collected. Total RNA was extracted using Concert ${ }^{\mathrm{Tu}}$ Plant RNA Reagent and treated with Turbo DNase-free (both from Invitrogen). The DNase treated RNA was reverse-transcribed using SuperScript ${ }^{\circ}$ III (Invitrogen) and Oligo-dT(18) primer. The abundance of CCA1 and TOC1 transcripts were estimated with qPCR and normalized to the abundance of $U B Q 10$ transcripts. The primers used for the qPCR are listed in Additional file 2: Table S6. 


\section{Additional files}

Additional file 1: Figure S1. Expression of PCFS4-TAP fusion protein in transgenic plants. The total protein extracts from the mutant (pcfs4-1) and the mutant containing the gene construct of 35S: PCFS4-TAP (PCFS4-TAP) were fractioned, blotted to membrane and immuno-detected using peroxidaseconjugated anti-peroxidase lgG against the TAP tag (upper-panel). The Coomassie blue stained gel image (lower-panel) showed an equal loading for the two samples. Figure S2. Verification of the PCFS4-TAP enrichment on the gene loci involved in circadian rhythm. Following the ChIP, the DNA abundance (mean \pm stdev) of the PCFS4-TAP enriched sites were determined using QPCR and normalized to the PCFS4-TAP enrichment on Tip41 (the control). Figure S3. PCFS4-TAP enrichment sites are associated with the sites where the alternative transcription or pre-mRNA processing occurs. The gene structures are represented by blue bars (light blue bars for $5^{\prime}$ and $3^{\prime}$ UTR) and lines (Gene model). The cDNAVESTs supporting the gene model are represented by green bars (CDNA/EST). The vertical black bars represent the PCFS4-TAP enrichment (Log fold enrichment) along the gene body. The red frames highlight the regions where the ES site are associated with alternative transcription or pre-mRNA processing supported by cDNA/ESTs.

Additional file 2: Table S1. The PCFS4-TAP enrichment site (ES) and their linked genes. Table S2. Enriched GO terms identified with GeneCodis and the genes within the GO terms. Table S3. Group of coherent GO terms generated through GeneTerm Linker. Table S4. Enriched GO terms identified using GOEAST. Table S5. ES-linked Genes within the enriched GO term "Regulation of Circadian Rhythm" (GO:0042752). Table S6. The primers used in this study.

\section{Competing interests}

The authors declare that they have no competing interests.

\section{Authors' contributions}

$\mathrm{QQL}$ and DX conceived the experiments; DX, RX, XY, DY carried out the experiments; DX, YW analyzed the data; DX and QQL wrote the manuscript. All authors read and approved the final manuscript.

\section{Acknowledgements}

The authors thank Hisashi Koiwa for gene constructs, other lab members for helpful discussions, and technical supports from the Center for Functional Genomics and Bioinformatics, Miami University. Special thanks to Lily Xing for language corrections of this manuscript. This work was funded in part by a grant from the US National Science Foundation (IOS-0817818) to QQL, a grant from Ohio Plant Biotech Consortium to DX and QQL, and additional supports from Miami University and Xiamen University. QQL received funding support from the Hundred Talent Plan of Fujian Province, China.

\section{Author details}

'Department of Botany, Miami University, Oxford, OH 45056, USA. ${ }^{2}$ Rice Research Institute, Fujian Academy of Agriculture Sciences, Fuzhou, Fujian, China. ${ }^{3}$ Key Laboratory of the Ministry of Education for Coastal and Wetland Ecosystems, and College of the Environment and Ecology, Xiamen University, Xiamen, Fujian 361102, China. ${ }^{4}$ Current Address: Department of Biology, Colorado State University, Fort Collins, CO 80526, USA. ${ }^{5}$ Current Address: Department of Medicinal Chemistry and Molecular Pharmacology, Purdue University, West Lafayette, IN, USA.

Received: 4 April 2013 Accepted: 27 August 2013

Published: 3 September 2013

\section{References}

1. Moore MJ, Proudfoot NJ: Pre-mRNA processing reaches back to transcription and ahead to translation. Cell 2009, 136:688-700

2. Birse CE, Minvielle-Sebastia L, Lee BA, Keller W, Proudfoot NJ: Coupling termination of transcription to messenger RNA maturation in yeast. Science 1998, 280:298-301.

3. Licatalosi DD, Geiger G, Minet M, Schroeder S, Cilli K, McNeil JB, Bentley DL: Functional interaction of yeast pre-mRNA 3 ' end processing factors with RNA polymerase II. Mol Cell 2002, 9:1101-1111.
4. Zhang Z, Fu J, Gilmour DS: CTD-dependent dismantling of the RNA polymerase II elongation complex by the pre-mRNA 3'-end processing factor, Pcf11. Genes Dev 2005, 19:1572-1580.

5. Zhang Z, Gilmour DS: Pcf11 is a termination factor in Drosophila that dismantles the elongation complex by bridging the CTD of RNA polymerase II to the nascent transcript. Mol Cell 2006, 21:65-74.

6. Amrani N, Minet M, Wyers F, Dufour ME, Aggerbeck LP, Lacroute F: PCF11 encodes a third protein component of yeast cleavage and polyadenylation factor I. Mol Cell Biol 1997, 17:1102-1109.

7. Klatt A, Zhang Z, Kalantari P, Hankey PA, Gilmour DS, Henderson AJ: The receptor tyrosine kinase RON represses HIV-1 transcription by targeting RNA polymerase II processivity. J Immunol 2008, 180:1670-1677.

8. Loya TJ, O'Rourke TW, Reines D: A genetic screen for terminator function in yeast identifies a role for a new functional domain in termination factor Nab3. Nucleic Acids Res 2012, 7:7.

9. Rougemaille M, Dieppois G, Kisseleva-Romanova E, Gudipati RK, Lemoine S, Blugeon C, Boulay J, Jensen TH, Stutz F, Devaux F, Libri D: THO/Sub2p functions to coordinate 3 '-end processing with gene-nuclear pore association. Cell 2008, 135:308-321.

10. Zhang Z, Klatt A, Henderson AJ, Gilmour DS: Transcription termination factor Pcf11 limits the processivity of Pol II on an HIV provirus to repress gene expression. Genes Dev 2007, 21:1609-1614.

11. Singh N, Ma Z, Gemmill T, Wu X, Defiglio H, Rossettini A, Rabeler C, Beane O, Morse RH, Palumbo MJ, Hanes SD: The Ess1 prolyl isomerase is required for transcription termination of small noncoding RNAs via the Nrd1 pathway. Mol Cell 2009, 36:255-266.

12. Ghazy MA, Gordon JM, Lee SD, Singh BN, Bohm A, Hampsey M, Moore C: The interaction of Pcf11 and Clp1 is needed for mRNA 3'-end formation and is modulated by amino acids in the ATP-binding site. Nucleic Acids Res 2012, 40:1214-1225.

13. Haddad R, Maurice F, Viphakone N, Voisinet-Hakil F, Fribourg S, MinvielleSebastia $L$ : An essential role for $C l p 1$ in assembly of polyadenylation complex CF IA and Pol II transcription termination. Nucleic Acids Res 2012, 40:1226-1239.

14. Holbein S, Scola S, Loll B, Dichtl BS, Hubner W, Meinhart A, Dichtl B: The Ploop domain of yeast Clp1 mediates interactions between CF IA and CPF factors in pre-mRNA 3' end formation. PLoS One 2011, 6:e29139.

15. Johnson SA, Kim H, Erickson B, Bentley DL: The export factor Yra1 modulates mRNA 3' end processing. Nat Struct Mol Biol 2011, 18:1164-1171.

16. Gross S, Moore C: Five subunits are required for reconstitution of the cleavage and polyadenylation activities of Saccharomyces cerevisiae cleavage factor I. Proc Natl Acad Sci U S A 2001, 98:6080-6085.

17. Hollingworth D, Noble CG, Taylor IA, Ramos A: RNA polymerase II CTD phosphopeptides compete with RNA for the interaction with Pcf11. Rna 2006, 12:555-560

18. Kim H, Erickson B, Luo W, Seward D, Graber JH, Pollock DD, Megee PC, Bentley DL: Gene-specific RNA polymerase II phosphorylation and the CTD code. Nat Struct Mol Biol 2010, 17:1279-1286.

19. Lunde BM, Reichow SL, Kim M, Suh H, Leeper TC, Yang F, Mutschler H, Buratowski S, Meinhart A, Varani G: Cooperative interaction of transcription termination factors with the RNA polymerase II C-terminal domain. Nat Struct Mol Biol 2010, 17:1195-1201.

20. Mayer A, Heidemann M, Lidschreiber M, Schreieck A, Sun M, Hintermair C, Kremmer E, Eick D, Cramer P: CTD tyrosine phosphorylation impairs termination factor recruitment to RNA polymerase II. Science 2012, 336:1723-1725.

21. Mayer A, Lidschreiber M, Siebert M, Leike K, Soding J, Cramer P: Uniform transitions of the general RNA polymerase II transcription complex. Nat Struct Mol Biol 2010, 17:1272-1278.

22. Seoane S, Lamas-Maceiras M, Rodriguez-Torres AM, Freire-Picos MA Involvement of Pta1, Pcf11 and a KICYC1 AU-rich element in alternative RNA 3'-end processing selection in yeast. FEBS Lett 2009, 583:2843-2848.

23. Xing D, Zhao H, Xu R, Li QQ: Arabidopsis PCFS4, a homologue of yeast polyadenylation factor Pcf11p, regulates FCA alternative processing and promotes flowering time. Plant J 2008, 54:899-910.

24. Zheng J, Xing D, Wu X, Shen Y, Kroll DM, Ji G, Li QQ: Ratio-based analysis of differential mRNA processing and expression of a polyadenylation factor mutant pcfs4 using arabidopsis tiling microarray. PLoS One 2011, 6:e14719.

25. Kim M, Ahn SH, Krogan NJ, Greenblatt JF, Buratowski S: Transitions in RNA polymerase II elongation complexes at the 3 ' ends of genes. Embo J 2004, 23:354-364 
26. Guo D, Hazbun TR, Xu XJ, Ng SL, Fields S, Kuo MH: A tethered catalysis, two-hybrid system to identify protein-protein interactions requiring post-translational modifications. Nat Biotechno/ 2004, 22:888-892.

27. Kang CH, Feng Y, Vikram M, Jeong IS, Lee JR, Bahk JD, Yun DJ, Lee SY, Koiwa H: Arabidopsis thaliana PRP40s are RNA polymerase II C-terminal domain-associating proteins. Arch Biochem Biophys 2009, 484:30-38.

28. Langmead B, Trapnell C, Pop M, Salzberg SL: Ultrafast and memory-efficient alignment of short DNA sequences to the human genome. Genome Biol 2009, 10:R25.

29. Ji H, Jiang $H, M a$ W, Johnson DS, Myers RM, Wong WH: An integrated software system for analyzing ChIP-chip and ChIP-seq data. Nat Biotechnol 2008, 26:1293-1300.

30. Czechowski T, Stitt M, Altmann T, Udvardi MK, Scheible WR: Genome-wide identification and testing of superior reference genes for transcript normalization in Arabidopsis. Plant Physiol 2005, 139:5-17.

31. Machanick P, Bailey TL: MEME-ChIP: motif analysis of large DNA datasets. Bioinformatics 2011, 27:1696-1697.

32. Tabas-Madrid D, Nogales-Cadenas R, Pascual-Montano A: GeneCodis3: a non-redundant and modular enrichment analysis tool for functional genomics. Nucleic Acids Res 2012, 40:W478-483.

33. Zheng Q, Wang XJ: GOEAST: a web-based software toolkit for Gene Ontology enrichment analysis. Nucleic Acids Res 2008, 36:W358-363.

34. McClung CR: The genetics of plant clocks. Adv Genet 2011, 74:105-139.

35. Nakamichi N: Molecular mechanisms underlying the Arabidopsis circadian clock. Plant Cell Physiol 2011, 52:1709-1718.

36. Lutz CS, Moreira A: Alternative mRNA polyadenylation in eukaryotes: an effective regulator of gene expression. Wiley Interdiscip Rev RNA 2011, 2:22-31.

37. Nilsen TW, Graveley BR: Expansion of the eukaryotic proteome by alternative splicing. Nature 2010, 463:457-463

38. Reddy AS, Shad Ali G: Plant serine/arginine-rich proteins: roles in precursor messenger RNA splicing, plant development, and stress responses. Wiley Interdiscip Rev RNA 2011, 2:875-889.

39. Xing D, Li QQ: Alternative polyadenylation and gene expression regulation in plants. Wiley Interdiscip Rev RNA 2011, 2:445-458.

40. Thomas PE, Wu X, Liu M, Gaffney B, Ji G, Li QQ, Hunt AG: Genome-Wide Control of Polyadenylation Site Choice by CPSF30 in Arabidopsis. Plant Cell 2012, 13:13.

41. Thomas PE, Wu X, Liu M, Gaffney B, Ji G, Li QQ, Hunt AG: Genome-wide control of poly(A) site choice by CPSF30 in Arabidopsis. Plant Cell 2012 24:4376-4388.

42. Kim Guisbert KS, Li H, Guthrie C: Alternative 3' pre-mRNA processing in Saccharomyces cerevisiae is modulated by Nab4/Hrp1 in vivo. PLoS Biol 2007, 5:e6.

43. Nedea E, He X, Kim M, Pootoolal J, Zhong G, Canadien V, Hughes T, Buratowski S, Moore CL, Greenblatt J: Organization and function of APT, a subcomplex of the yeast cleavage and polyadenylation factor involved in the formation of mRNA and small nucleolar RNA 3'-ends. J Biol Chem 2003, 278:33000-33010.

44. Deng X, Gu L, Liu C, Lu T, Lu F, Lu Z, Cui P, Pei Y, Wang B, Hu S, Cao X: Arginine methylation mediated by the Arabidopsis homolog of PRMT5 is essential for proper pre-mRNA splicing. Proc Natl Acad Sci U S A 2010, 107:19114-19119.

45. James AB, Syed NH, Bordage S, Marshall J, Nimmo GA, Jenkins Gl, Herzyk P, Brown JW, Nimmo HG: Alternative splicing mediates responses of the Arabidopsis circadian clock to temperature changes. Plant Cell 2012, 24:961-981.

46. Petrillo E, Sanchez SE, Kornblihtt AR, Yanovsky MJ: Alternative splicing adds a new loop to the circadian clock. Commun Integr Biol 2011, 4:284-286.

47. Sanchez SE, Petrillo E, Beckwith EJ, Zhang X, Rugnone ML, Hernando CE, Cuevas JC, Godoy Herz MA, Depetris-Chauvin A, Simpson CG, et al: A methyl transferase links the circadian clock to the regulation of alternative splicing. Nature 2010, 468:112-116.
48. He Y, Michaels SD, Amasino RM: Regulation of flowering time by histone acetylation in Arabidopsis. Science 2003, 302:1751-1754.

49. Johnson L, Cao X, Jacobsen S: Interplay between two epigenetic marks. DNA methylation and histone H3 lysine 9 methylation. Curr Biol 2002, 12:1360-1367.

doi:10.1186/1471-2164-14-598

Cite this article as: Xing et al.: The regulatory role of Pcf11-similar-4 (PCFS4) in Arabidopsis development by genome-wide physical interactions with target loci. BMC Genomics 2013 14:598.

\section{Submit your next manuscript to BioMed Central and take full advantage of:}

- Convenient online submission

- Thorough peer review

- No space constraints or color figure charges

- Immediate publication on acceptance

- Inclusion in PubMed, CAS, Scopus and Google Scholar

- Research which is freely available for redistribution

Submit your manuscript at www.biomedcentral.com/submit
C) BioMed Central 